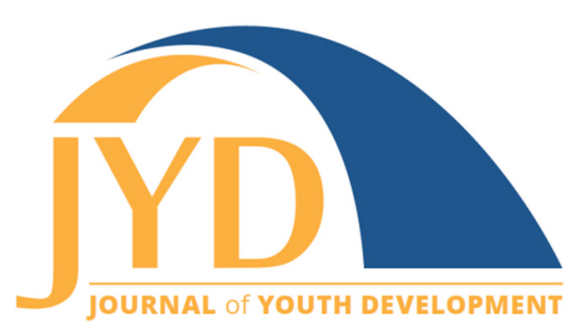

http://jyd.pitt.edu/ | Vol. 13 Issue 1-2 DOI 10.5195/jyd.2018.537 | ISSN 2325-4017 (online)

\title{
Striking a Balance: An Exploration of Staff-Camper Relationship Formation
}

\author{
Rachel O. Rubin \\ University of Massachusetts Boston \\ rachel.rubin001@umb.edu
}

Matthew Hagler

University of Massachusetts Boston

matthew.hagler001@umb.edu

Samantha A. Burton

University of Massachusetts Boston

samantha.burton001@umb.edu

Jean E. Rhodes

University of Massachusetts Boston

jean.rhodes@umb.edu

\section{Abstract}

Previous research highlights a range of positive developmental outcomes associated with attending summer camp. Close staff-camper relationships likely contribute to positive development, but little is known about how these bonds are formed. The current study utilized constructivist thematic analysis of interviews with campers ( $\mathrm{n}=8$ ) and staff members $(\mathrm{n}=7)$ at an overnight summer camp to examine the factors and processes that promote or inhibit close staff-camper relationship formation. The main themes identified were striking a balance, level of experience, and relationship-promoting behaviors. Staff members experience apparent paradoxes in their roles (e.g., relating to campers while also exerting authority), but navigate these tensions by using relationship-promoting behaviors and through increased experience. These findings suggest that staff training and supervision should emphasize relationshippromoting behaviors, continue throughout the summer, and be informed by campers' perspectives. Additionally, camp administrators should capitalize on accrued experience by prioritizing staff retention.

Key words: summer camp, youth-adult relationships, mentoring, qualitative

(cc) EY New articles in this journal are licensed under a Creative Commons Attribution 4.0 License. This journal is published by the University Library System, University of Pittsburgh and is cosponsored by the University of Pittsburgh Press. The Journal of Youth Development is the official peer-reviewed publication of the National Association of Extension 4-H Agents and the National AfterSchool Association. 


\section{Staff-Camper Relationship Formation}

Over 10 million children attend summer camp each year (American Camp Association (ACA), 2006). Research has highlighted a range of positive developmental outcomes associated with attending summer camp (Bialeschki, Henderson, \& James, 2007; Thurber, Scanlin, Scheuler, \& Henderson, 2007). Less is known, however, about the "active ingredients" of the camp experience (i.e., the specific aspects that foster positive developmental outcomes) such as the close and supportive staff-camper relationships often forged. The current study utilized thematic analysis of interviews with campers and counselors at an overnight summer camp to examine the factors and processes that contribute to close staff-camper relationship development as well as understanding the barriers to forming these connections.

\section{Background}

Summer camps represent fertile ground for the formation of strong ties between campers and staff members. The unique structure of camp, particularly residential settings, affords rich opportunities for youth and caring adults to engage in informal conversations and mutually enjoyable activities that give rise to close bonds (ACA, 2006). Counselors can provide a safe, supportive context while transmitting values and guidance. Moreover, because many camp counselors are relatively close in age to campers and were campers themselves, they are wellpositioned to connect with their campers. Beyond emotional support, camp counselors are often prepared to provide coaching and life skills that build on campers' strengths (Garst \& Johnson, 2005; Snider \& Farmer, 2016).

Positive Youth Development (PYD) theory holds that youth thrive when their strengths are aligned with contextual assets (Lerner, 2005). Camp counselors and other staff members represent key contextual assets (Scales, Benson, \& Mannes, 2006). In a large survey of youth participants in ACA-accredited camps throughout the United Sates, nearly $70 \%$ of campers reported receiving optimal levels of support from adults at camp, compared to an average $40 \%$ in other youth organizations (e.g., after-school programs) (ACA, 2006). In fact, many camp counselors view themselves as mentors who encourage, teach, and listen to youth (Garst \& Johnson, 2005). In a qualitative study, Snider and Farmer (2016) interviewed young adults who spent several years as both campers and counselors. These participants spoke more enthusiastically about staff-camper relationships than any other topic, and the researchers concluded that these bonds were the most critical determinant of campers' positive developmental experiences. 


\section{Staff-Camper Relationship Formation}

Although these studies highlight the importance of relationships, few have explored relationship-promoting factors and processes. A review of the literature of other adult-youth relationships (i.e., teacher-student, coach-athlete, and adult-youth mentoring) suggests that adults' self-efficacy and intentionality in building relationships, shared interests, and respect for youth autonomy promote the formation of high-quality bonds (e.g., Ehrlich, Deutsch, Fox, Johnson, \& Varga, 2016; Jackson, Grove, \& Beauchamp, 2010; Mashburn, Hamre, Downer, \& Pianta, 2006; Norman \& French, 2013; Raposa, Rhodes, \& Herrera, 2016). However, given the proximity of age between campers and counselors, these relationships might best be approximated in studies of cross-age peer mentoring programs, which create matches between older and younger adolescents. This small but growing body of literature also emphasizes the importance of building a connection between mentor and mentee as the key ingredient in promoting youth development (Karcher, 2013). Additionally, peer mentoring studies emphasize mentors' understanding of teen culture and being seen as credible by mentees as well as the mutual psychosocial development of both mentor and mentee (Grossman \& Bulle, 2006). Because peer mentors are still undergoing their own development, they too might be actively honing competencies while working with younger youth (Karcher, Davis, \& Powell, 2002), which is likely true of camp counselors.

\section{The Current Study}

In summary, camps promote positive youth development. Although research suggests that staff-camper bonding is important, most studies have focused on youth outcomes or participants' retrospective perceptions of these relationships. The processes through which these relationships form, and the factors that promote them, are relatively unknown.

With these gaps in mind, the current study utilized qualitative methodology to examine the processes underlying the formation of close staff-camper relationships, as well as barriers that interfere with close relationship formation. We utilized constructivist thematic analysis of camper and staff interviews to generate themes that address the following research questions: (a) what camper, counselor, and contextual factors promote staff-camper bonding, and (b) what are the processes through which these factors produce close relationships? These themes are presented with representative quotes, contextualized in existing literature, and used to make recommendations for practice and policy. 


\section{Staff-Camper Relationship Formation}

\section{Method}

Data were collected at a Jewish overnight summer camp in the Midwest serving children 7 to 15 years of age. This camp opened in the early 1900s with a mission of offering youth opportunities to grow, acquire skills, and have fun in the context of a strong, supportive community. Although Jewish values are infused in camp programming (e.g., weekend services, prayers at meals, Jewish educational activities, and the use of Hebrew words), the camp also offers a wide range of general activities such as sports, drama, arts and crafts, horseback riding, and boating.

\section{Participants}

Participants were rising sixth-grade through ninth-grade campers and their staff members. Participants included eight campers ( $50 \%$ female, $M_{a g e}=12.75, S D=.89$ ) and seven staff members ( $57 \%$ female, $\left.M_{a g e}=18.86, S D=1.21\right)$. Of the seven staff members, four were returning staff and three were first time staff members (see Table 1). All youth were returning campers (see Table 2). All participants gave informed consent or assent; parents of all minors provided consent. In addition, pseudonyms are used to maintain confidentiality.

Table 1. Staff Member Characteristics

\begin{tabular}{|llll|}
\hline Staff member & Year on staff & Gender & Age \\
\hline Aaron & First & Male & 18 \\
Danielle & Fourth & Female & 20 \\
Eric & First & Male & 17 \\
Leslie & Third & Male & 18 \\
Naomi & Fourth & Female & 20 \\
Stephanie & Third & Female & 19 \\
\hline
\end{tabular}


Table 2. Camper Characteristics

\begin{tabular}{|llll|}
\hline Camper & Year at camp & Gender & Age \\
\hline Ariella & Fourth & Female & 13 \\
Brandon & Second & Male & 12 \\
Emily & Seventh & Female & 14 \\
James & Third & Male & 11 \\
Laura & Sixth & Female & 13 \\
Lucas & Sixth & Male & 13 \\
Sarah & Seventh & Female & 13 \\
Will & Third & Male & 13 \\
\hline
\end{tabular}

\section{Procedure}

The first author completed data collection while living and working at camp, fully immersing herself in the context. She was an insider at this camp, having been a camper and staff member for several years. The implications of this insider role are addressed in the discussion section.

\section{Recruitment}

Parents received an email from camp administration explaining the study, the camp's approval of the research, and a link to consent forms for parents to read and fill out online. On the first day of camp, the researcher handed out assent forms to campers whose parents had consented. For staff member recruitment, the researcher handed out paper consent or assent forms in person during staff week.

\section{Interviews}

Among the pool of consenting staff and campers, the first author requested information from supervisors about individuals whose relationships were indicative of a range of strengths and difficulties. That is, supervisors were asked to identify staff and campers who tended to form positive, close bonds, as well as staff and campers who tended to have difficulty forming connections, in order to capture both relationship-promoting and -inhibiting factors. Interviews 


\section{Staff-Camper Relationship Formation}

ranged from 15-60 minutes. On average, camper interviews lasted 25 minutes, and staff member interviews lasted 45 minutes. All participants were compensated with a $\$ 10$ Amazon gift card.

Interviews took place on camp grounds in June and July of 2016 during the last two weeks of the first 4-week camp session, giving staff-camper relationships time to form. The first author created and utilized a semi-structured interview protocol, while also employing a relational interview approach in which she followed the participants' lead (Josselson, 2013). The protocol included questions about experiences at summer camp, with a focus on factors and processes that promote or obstruct the formation of staff-camper relationships. The first author used multiple techniques to ensure that saturation was reached and therefore an appropriate sample size was used. These techniques included examining researcher lens and interviewing a diverse sample of participants (Fusch \& Ness, 2015). In addition, when no new concepts related to the research aims emerged during several consecutive interviews, the researchers determined saturation was reached. Interviews were audio recorded and transcribed verbatim.

\section{Data Analysis}

We used constructivist thematic analysis to explore the processes through which close relationships between campers and staff are built, as well as barriers to relationship formation (Braun \& Clarke, 2006). Additionally, we used insights from the first author's immersion in the camp setting, such as observation of participants, to inform coding. The first author read each of the 15 interviews and developed a codebook grounded in iterative analysis of the data. The codebook consisted of in vivo codes (e.g., "being around") as well as descriptive codes (e.g., staff to camper differential treatment) (Saldana, 2012). We fine-tuned the codebook by adding, removing, and broadening codes when deemed appropriate (Braun \& Clarke, 2006).

We coded the first four interviews together, discussing discrepancies until consensus was reached. The remaining interviews were divided, with the first author coding all interviews and the second and third authors each coding half. Coding pairs met regularly to discuss discrepancies and reach consensus. After coding, we met several times to discuss connections between codes, search for repeated patterns of meaning, and form overarching themes (Braun \& Clarke, 2006). After developing themes, we returned to the original data to check the themes for representativeness. 


\section{Staff-Camper Relationship Formation}

We employed various techniques to ensure analytic rigor. Memoing was utilized throughout data collection and analysis to aid in the development of themes (Riessman, 2008; Strauss \& Corbin, 1998). Additionally, investigator responsiveness was maintained to remain open during the analysis process and relinquish poorly supported ideas (Morse, Barrett, Mayan, Olson, \& Spiers, 2002). Finally, we used the verification strategy of thinking theoretically to regularly check and recheck emerging ideas (Morse et al., 2002).

\section{Results}

Through thematic analysis, we identified several themes and subthemes. The three overarching themes are striking a balance, level of experience, and relationship-promoting behaviors. Each theme is discussed below.

\section{Striking a Balance}

The striking a balance theme captures staff members' perceptions of tension in their role, such as how to interact with different campers, draw boundaries with campers, and prioritize time and energy.

\section{Differential Treatment}

The first subtheme of striking a balance is differential treatment, which represents decisions about how to interact with each camper. Some staff members discussed ways in which they combatted inherent preferences for certain campers and tried to treat each camper equally. For instance, Leslie expressed, "I feel like it's really okay to have favorite campers but you just have to make sure that after you give attention to that camper you go on to the next camper. And like in my head I always...make sure I sit next to someone new at every meal or...sit at night time at someone else's bed." Although Leslie acknowledged having favorite campers, she discussed actively striving for equal treatment.

At the same time, staff members discussed taking different approaches with campers based on individual needs and characteristics. Danielle explained that "every camper is on a different plan," and described giving some campers (e.g., one who struggled with emotion regulation) more attention or breaks from activities during difficult moments. Although several other staff members discussed taking an individualized approach, both staff and campers recognized the pitfalls of giving certain campers more attention. For instance, one camper, Sarah, noted that a 


\section{Staff-Camper Relationship Formation}

major barrier to forming close relationships with counselors was "campers who like take up all of [staff members'] time 'cause they're complicated." Although Sarah acknowledged that the attention some campers receive is not necessarily favoritism, she maintained that differential treatment hindered campers' abilities to connect with staff members. Several staff members echoed this dilemma.

Although staff members were thoughtful about campers' reactions to differential treatment, notable inconsistencies existed between campers' and staff's perceptions. Counselor Aaron discussed liking some campers more than others, but said, "I don't show it to them. I treat them the same way...so it doesn't...get in the way of the camp experience." However, camper Brandon described another camper as "really small, popular, funny, and all that. [Staff members] really like him," which made Brandon feel "left out." While Aaron and other counselors believed they did not show favoritism, Brandon's remarks suggest that campers may perceive more than counselors realize.

\section{Role Negotiation}

The second subtheme, role negotiation, captures how staff members navigate boundaries. Staff members held different beliefs about how authoritative to be, how to discipline campers, and how much personal information to disclose. Campers also demonstrated different perspectives on staff members' boundary-setting or boundary-crossing behaviors.

Many staff members discussed tension between being a friend and an authority figure. One factor affecting staff members' decisions about boundaries was camper age. For instance, counselor Leslie noted that "you don't really need to be an authority figure, you just need to be more of like a role model, or like a mentor" for teenage campers. She went on to say that her campers "felt like they could tell me their drama, their gossip, and they knew that it wasn't going to get spread around, but also I was going to say my opinion...if I thought it was mean or something." This staff member sought to offer her insight as a role model without being overly authoritative. Similarly, when sharing information with campers, Stephanie used her understanding of campers' developmental stage to inform decisions about boundary negotiation: "They're not like the younger ones so...they do have maturity, and they're at this stage where they do want to know stuff." Here, Stephanie was willing to stretch her boundaries and be more honest with older campers. 


\section{Staff-Camper Relationship Formation}

Staff members also considered situational factors when balancing roles, and discussed knowing the time and place to have fun or assert authority. For instance, Aaron claimed that his campers respected him and his co-staff "because we're funny with [campers], but we also...know when the right time to be serious is." Similarly, Danielle explained that being able to "have fun with [campers] and...rewarding them when they're doing the right thing" leads to a mutually respectful relationship in which campers "listen to [staff] when...you're telling them that they made... a choice that wasn't the right choice." Although some staff members described an ability to walk this line, others commented on the difficulty of striking a balance. For instance, Stephanie described her relationship with one camper in which "it's getting to the point where she thinks we're...best friends....she's very like hands on, touchy-feely...and I've told her,...'No, I'm not joking.' Like, we have to have...that boundary." This first-time staff member realized that she had become too friendly with a camper and wanted to reestablish appropriate boundaries.

Most campers preferred when staff members were "more like a camper than a staff" and less "like an authority." They discussed that closer staff-camper relationships formed when staff members "talk to us like friends" and "act like kids." They often highlighted staff transparency and self-disclosure. For instance, Emily preferred when staff members "are open about their feelings 'cause they like [it] when we are" and disliked when staff members "try to hide it" because "[campers] can see they're upset." This camper believed that hiding emotions threatens authenticity in relationships.

Further, a general consensus was found among campers that staff members' exertion of authority, particularly by yelling, made campers less likely to bond with staff. For instance, Laura asserted that it "isn't really helping" when staff members yell at campers to get ready, and Ariella said they "should've just listened and not really yelled" at campers during tough moments. Lucas said his favorite time at camp was at night in the cabin, when "[staff members] are with us...they're one of us....We just talk...they don't yell at us to do anything. We do what we want to do. They do it with us."

However, campers also valued the mentorship that distinguishes staff members from same-age peers. For example, Emily noted how nice it was that staff give advice because "it makes us feel like they're an older sister, not even a mom...they're like our older best friend." Here, friendship with staff members is characterized by a sense of respect and guidance. Further, James expressed a preference for staff members to use some level of discipline, likening his favorite staff member to "a good parent that has fun and everything but if you step on a line he puts 


\section{Staff-Camper Relationship Formation}

you back in line." For this camper, having counselors who enforced rules and boundaries facilitated closer staff-camper relationships.

\section{Priorities}

The final subtheme, priorities, highlights how staff members choose to use their finite time and energy at camp. This subtheme explores the extent to which staff members balance the needs of their campers with other responsibilities and desirable activities. Both staff and campers discussed the need for staff to balance the time they spend with campers and the time they spend with other staff members. Some staff members, like Naomi, felt it was "pretty easy" to navigate this balance: "During the day I'm supposed to be with campers and that's when I'm...making camper relationships,....and then you...have the nighttime to be with your friends and maintain those relationships." However, other staff members, such as Eric, explicitly described struggling with this balance: "I feel like I personally have trouble balancing the staff relationships with the camper relationships, and I feel like I'm not alone in that." Eric explained that his interactions with female staff during the camp day "can be a distraction," occasionally taking his attention from campers.

Some staff members emphasized that campers notice when staff-staff relationships are being prioritized and consequently feel underappreciated. For example, David stated that campers are "going to see....whether you're putting the campers first or whether you...talk to counselors the whole day....Every kid wants to feel like they're wanted, that people care about them....If that's not there, they're not gonna have a great relationship with you." Campers echoed this sentiment. For instance, Emily noted that campers "can't get as close with them [staff members] cause they're busy with their friends."

Campers also discussed their ability to discern staff members' priorities. For example, Laura perceived a staff members' lack of interest, noting, "when she talked to me...she wasn't rude but like, you could kind of tell that she would rather be doing something else." On the other hand, campers expressed appreciation when staff members made efforts to spend time with them. For example, Brandon described how he particularly liked one counselor who "decided to come to [the campers'] tree hut" during his period off "even though he wasn't supposed to," making it clear that he was putting his campers first. Even so, some campers recognized that staff members had competing demands and limited time and energy, and should have time to socialize with each other. Sarah said, "I think it's fair because they should be able to hang out 
Journal of Youth Development | http://jyd.pitt.edu/ | Vol. 13 Issue 1-2 DOI 10.5195/jyd.2018.537

\section{Staff-Camper Relationship Formation}

with their friends. But they should also...hang out with...their campers."

\section{Level of Experience}

The level of experience theme captures the extent to which staff members' prior experiences impacted relationships with campers. Counselors and campers described how previous familiarity and interactions, both in and outside of camp, facilitated stronger relationships. Additionally, staff described how their own experience level in the staff member role contributed to their ability to manage responsibilities and connect with campers.

\section{Previous Connections}

The first subtheme of level of experience is previous connections, which represents staffcamper bonding that occurred during previous camp sessions or outside of camp. These preexisting connections facilitated the cultivation and maintenance of close relationships. Eric, a returning counselor, described a repeat camper with whom other counselors were struggling to connect: "People have been telling me how bad he is, and...he's so good for me. We started when he was seven years old here, and I was his first counselor...and I had him again the next year....so I feel like he was very comfortable with me." Campers similarly described having closer relationships with staff members whom they knew in previous camp sessions or other contexts. For instance, camper James noted that he felt particularly close to a counselor he knew before camp: "I just hung out with him sometimes with my cousins before I went to camp... and...the second night of camp I was feeling homesick, and he took me outside and helped me." Multiple campers and staff members highlighted how prior interactions and relationships brought them closer together in the camp context.

\section{Competence}

The second subtheme of level of experience is competence, which captures the impact of staff members' cumulative experiences as counselors. Often, staff members reflected on the challenges of their first year on staff. As a first-time staff member, Stephanie described her own difficulties knowing camp rules, saying, "I think a barrier to forming the relationship is...as a first-year staff member, I was cautious about where the boundaries would be. And if I could tell the kids this, if I could tell them that, and...I was kind of standoffish." Stephanie's lack of confidence and competence as a counselor negatively affected her connections with campers. Similarly, Eric, a returning staff member, compared first year and returning staff members' 


\section{Staff-Camper Relationship Formation}

responses to camper misbehavior, saying "I could see where first time staff members are doing things wrong...they didn't really know how camp sort of goes....I just keep my cool." Here, Eric points out how additional experience builds knowledge about how to respond to campers effectively.

\section{Relationship-Promoting Behaviors}

The relationship-promoting behaviors theme captures specific staff behaviors that strengthened staff-camper relationships. These tangible efforts and actions were key to initiating and cultivating connections, regardless of experience level and difficulties striking a balance.

Several campers and staff discussed the importance of staff making themselves available, just "being around." For example, counselor David, said, "I'm just always around....If [campers] need to come and talk to me I'm near." This staff member emphasized being available, rather than initiating conversations: "I'm not gonna go walking up to [a specific camper], but I'm gonna be around the group." Other staff members highlighted the importance of initiating connections with campers. Leslie said, "I just...really try to make sure that I talk to every single camper every single day....like, 'How did you sleep? How was your day?...What did you have at lunch?'...I think as long as you have a conversation with every single camper every single day that's meaningful, you build the relationship naturally." Campers noted how an active approach facilitated relationship-building. For example, Laura described staff members she did not connect with: "They just don't...branch out...they don't start the conversation. They'll like talk to you but only if you talk to them first....It's kind of awkward." This camper found it easier to connect with staff members who initiated interactions.

Staff members further emphasized the importance of making individualized connections with campers, even those with differing interests. For example, Danielle said she connects with campers by "showing interest in their own interests....I'm not interested in basketball, but I had campers who loved playing, so I'd play with them." This staff member described a youthcentered approach, focused on engaging with campers through activities they enjoy. In contrast, camper Brandon described a staff member who only "likes doing all that sports stuff, but when it comes to talking and relaxing, not him.... When we're not doing anything he lays there....with his phone all day." This staff member only engaged with campers during activities that were of interest to him, which limited his ability and opportunity to connect with campers. 


\section{Staff-Camper Relationship Formation}

In addition to general efforts to be available and initiate connections, participants discussed moments when staff members provided help or assistance. James described a particularly observant staff member who engaged him when he was feeling homesick or isolated: "He'd always interrupt me when I just was looking like I was just thinking and sitting alone.....He said, 'You wanna go do something fun and help me, you wanna help me gather sticks?' I said 'Yeah."' More generally, Emily discussed how her favorite counselor's help and advice facilitated trust and closeness in their relationship: "It's just on such an intimate level...I feel like I can tell her anything and I know that she'll help me through it."

\section{Discussion}

The current study examined camper and staff member narratives in order to explore the processes through which staff members and campers form close connections at camp, as well as factors that hinder these relationships. Our findings reveal a number of individual, contextual, and relational factors and processes that influence staff-camper relationships. This research fills an important gap in the extant body of camp literature, which has primarily focused on outcomes rather than processes, and informs tangible recommendations for camp practice and policy that aim to enhance the prevalence, quality, and equitable distribution of these important bonds.

Staff members were often challenged to strike the optimal balance across several domains. They discussed trying to treat all campers equally while taking an individualized approach, establish rapport while maintaining authority, and prioritize campers while fulfilling other responsibilities and self-care. Many recognized that there is a "time and a place" for certain approaches, and highlighted the importance of flexibility and dimensionality in their roles.

Both staff and campers spoke against explicit favoritism, and emphasized the importance of staff forming relationships with every camper. However, because campers vary in their needs, interests, and personalities, connecting with each camper may not always appear 'equal.' Staff members struggled to connect with all campers, while providing extra time and attention to campers who needed it.

Consistent with research on teacher-student relationships (Davis, 2006), youth expressed wanting relationships with staff marked by respect, collaboration, and authenticity. This idea meant using humor, self-disclosure, and tolerance to form relationships that, in many ways, were friendships. Still, all staff, and even some campers, recognized the importance of 


\section{Staff-Camper Relationship Formation}

maintaining boundaries. This recognition meant occasionally exerting authority, but more often offering wisdom and guidance. Finally, participants universally endorsed the belief that campers should be staff members' top priority. Yet, many campers recognized that staff members had finite time and energy, and forgave moments of temporary disengagement.

Staff members described struggling with these balancing acts in the past and present. Fortunately, managing these difficulties seemed to get easier with time and experience. Returning staff members felt more competent and confident in their roles, as well as more comfortable sitting with ambiguity and maintaining flexibility. Seemingly, they developed a stronger sense of self-efficacy, which has been shown to promote high-quality adult-youth relationships (Raposa et al., 2016; Yoon, 2002). Further, both campers and staff members spoke of the trust, comfort, and rapport that grew through repeated, deepening connections over multiple years. These results reflect the importance of attending to both campers' and staff members' development, which is emphasized in literature on cross-age peer mentoring (Karcher, 2013).

Our results highlight several relationship-promoting behaviors that facilitated close connections with campers, even in the face of inexperience. Our findings echo results from mentoring contexts in which youth developed closer relationships with adults who invested time and attention, initiated connections, and made an active effort to understand them (Ehrlich et al., 2016). Campers recognized and reciprocated staff members' desire and intentionality to engage with them, leading to reciprocal, co-created connections.

The relationship-promoting and -inhibiting behaviors identified by our participants align closely with the principles of positive youth development (PYD), which emphasize the importance of adults prioritizing relationships with youth, displaying genuine interest and engagement, and demonstrating intentionality in forming connections. Conversely, actions that suggest disinterest, disengagement, or passivity in connecting with youth (e.g., prioritizing staff-staff relationships, spending free time away from youth sleeping or using cell phones, waiting for youth to initiate connections) stand in contrast to PYD principles of active adult engagement (Ehrlich et al., 2016; Grossman \& Bulle, 2006; Scales et al., 2006)

\section{Practical Implications}

These findings can inform camp programming and promote positive youth development. First, our themes can directly inform staff training and ongoing supervision of camp staff. For 


\section{Staff-Camper Relationship Formation}

example, relationship-promoting behaviors (e.g., initiating connections, being present) should be emphasized in relationship-building training modules. However, pre-camp training is not the only avenue for improvement. Staff members discussed difficulties forming individual connections while maintaining equitable treatment, negotiating boundaries, and prioritizing camper relationships, and some staff members noted improvement in these areas through corrective experiences. Training leaders and supervisors can capitalize on experiential learning opportunities by facilitating ongoing dialogues about these challenges. For instance, camp leaders can identify times, such as at night, or during meals or activities, when a rotating subset of staff can engage in additional training, discussions, and supervision.

Further, insights gained from camper interviews illustrate the importance of youth involvement in staff training. For example, several staff members expressed the belief that campers were not aware of underlying favoritism, but campers' remarks suggested that campers perceive more than staff members realize. Thus, providing opportunities for campers to share their positive or negative experiences forming relationships with staff could be useful additions to staff trainings.

Finally, our findings on the importance of staff members' level of experience suggest that camp administrators should prioritize staff retention. Greater resources allocated to retention, such as opportunities for professional development, advancement, and increased pay, could incentivize staff to return for subsequent years. New staff members may benefit from more explicit mentoring with experienced co-staff, who can guide them through the challenges their first year and provide insights on how to best manage their roles and responsibilities while creating meaningful connections with campers. Further, many campers and counselors identified their closest relationships as those that recurred over multiple camps sessions and years. Administrators might consider having staff members "follow" particular cohorts of campers, allowing them to establish strong foundations of trust that they build on year after year.

\section{Strengths, Limitations, and Future Directions}

Although our findings have several important practical implications, they should be interpreted in the context of certain limitations. First, as an insider, it is possible that the first author made assumptions and missed nuances due to overfamiliarity with the context (Bonner \& Tolhurst, 2002). To counteract these risks, she wrote memos while living at camp, critically examined her assumptions, and included two outside researchers in the analytic process. Additionally, 


\section{Staff-Camper Relationship Formation}

because of her dual roles, she ensured that participants understood her new role as researcher, the confidentiality of their responses, and their ability to withdraw at any time (Labaree, 2002).

This study was conducted exclusively at a Jewish overnight camp, which may limit the generalizability of its findings. For example, participants' shared faith and culture, previous connections through the wider religious community, and the camp's commitment to Jewish values (e.g., community, welcoming guests) likely influenced the nature and quality of staffcamper relationships. Future studies should examine the role of religious values in camps and compare staff-camper relationships across faith-based and secular camps. To further assess and enhance generalizability, future research should be conducted in a diverse selection of camps or in camps serving more diverse youth populations. Another potential limitation is that participants were selected based on supervisor-reported tendencies to have close or distant staff-camper relationships and all campers were returning campers. Thus, the sample may differ from the general camp population.

With these limitations in mind, this study has several strengths. First, data collection and analysis utilized rigorous qualitative methods, which allowed for a deep, nuanced understanding of the factors and processes through which staff members and campers form connections. Additionally, this study used both staff member and camper perspectives. Much of the existing research on adult-youth relationships (e.g., teacher-student, mentor-mentee) emphasizes adult perspectives without giving adequate attention to the youth perspective. Here, youths' voices were highlighted in both data collection and analysis. Further, the present study's examination of the contribution of contextual assets (e.g., supportive staff members) to youth development was grounded in a strengths-based PYD approach rather than taking a deficit-based perspective to campers' difficulties or misbehaviors. The camp context offers a unique opportunity to facilitate strong adult-youth connections and positive youth outcomes; increasing our understanding of these relationships and applying evidence-based techniques to cultivate them more intentionally are important next steps in camp research and practice.

\section{Acknowledgements}

The authors acknowledge funding from the MacArthur Foundation Research Network on Connected Learning and MENTOR: The National Mentoring Partnership. The second author is also supported by a National Science Foundation Graduate Research Fellowship. Finally, we would like to thank the campers, staff members, and camp administrators for their participation in and/or support of this project. 
Journal of Youth Development | http://jyd.pitt.edu/ | Vol. 13 Issue 1-2 DOI 10.5195/jyd.2018.537 Staff-Camper Relationship Formation

\section{References}

American Camp Association. (2006). Inspirations: Developmental supports and opportunities of youths' experiences at camp. Martinsville, IN: American Camp Association.

Bialeschki, M. D., Henderson, K. A., \& James, P. A. (2007). Camp experiences and developmental outcomes for youth. Child and Adolescent Psychiatric Clinics of North America 16(4), 769-788. doi: 10.1016/j.chc.2007.05.011

Bonner, A., \& Tolhurst, G. (2002). Insider-outsider perspectives of participant observation. Nurse Researcher, 9(4), 7-19. doi: 10.7748/nr2002.07.9.4.7.c6194

Braun, V., \& Clarke, V. (2006). Using thematic analysis in psychology. Qualitative Research in Psychology, 3(2), 77-101. https://doi.org/10.1191/1478088706qp063oa

Davis, H. A. (2006). Exploring the contexts of relationship quality between middle school students and teachers. The Elementary School Journal, 106(3), 193-223. doi: 10.1086/501483

Ehrlich, V. A., Deutsch, N. L., Fox, C. V., Johnson, H. E., \& Varga, S. M. (2016). Leveraging relational assets for adolescent development: A qualitative investigation of youth-adult "connection" in positive youth development. Qualitative Psychology, 3(1), 59-78. doi: 10.1037/qup0000046

Fusch, P. I., \& Ness, L. R. (2015). Are we there yet? Data saturation in qualitative research. The Qualitative Report, 20(9), 1408-1416.

Garst, B., \& Johnson, J. (2005). Adolescent leadership skill development through residential 4-H camp counseling. Journal of Extension, 43(5).

Grossman, J. B., \& Bulle, M. J. (2006). Review of what youth programs do to increase the connectedness of youth with adults. Journal of Adolescent Health, 396), 788-799.

doi: 10.1016/j.jadohealth.2006.08.004

Jackson, B., Grove, J. R., \& Beauchamp, M. R. (2010). Relational efficacy beliefs and relationship quality within coach-athlete dyads. Journal of Social and Personal Relationships, 278), 1035-1050. doi: $10.1177 / 0265407510378123$

Josselson, R. (2013). Interviewing for qualitative inquiry: A relational approach. New York, NY: Guilford Press.

Karcher, M. J. (2013). Cross-age peer mentoring. In Handbook of Youth Mentoring (pp. 233-258). doi: 10.4135/9781412996907.n16

Karcher, M. J., Davis, C., \& Powell, B. (2002). The effects of developmental mentoring on connectedness and academic achievement. The School Community Journal, 12(2), 35-50.

Labaree, R. V. (2002). The risk of "going observationalist": Negotiating the hidden dilemmas of being an insider participant observer. Qualitative Research, 2(1), 97-122.

doi: $10.1177 / 1468794102002001641$ 
Journal of Youth Development | http://jyd.pitt.edu/ | Vol. 13 Issue 1-2 DOI 10.5195/jyd.2018.537

Staff-Camper Relationship Formation

Lerner, R. M. (2005). Positive youth development: A view of the issues. The Journal of Early Adolescence, 25(1), 10-16. doi: 10.1177/0272431604273211

Mashburn, A. J., Hamre, B. K., Downer, J. T., \& Pianta, R. C. (2006). Teacher and classroom characteristics associated with teachers' ratings of prekindergartners' relationships and behaviors. Journal of Psychoeducational Assessment, 24(4), 367-380. doi: 10.1177/0734282906290594

Morse, J. M., Barrett, M., Mayan, M., Olson, K., \& Spiers, J. (2002). Verification strategies for establishing reliability and validity in qualitative research. International Journal of Qualitative Methods, 1(2), 13-22. doi: 10.1177/160940690200100202

Norman, L., \& French, J. (2013). Understanding how high performance women athletes experience the coach-athlete relationship. International Journal of Coaching Science, 71 ), 3-24.

Raposa, E. B., Rhodes, J. E., \& Herrera, C. (2016). The impact of youth risk on mentoring relationship quality: Do mentor characteristics matter? American Journal of Community Psychology, 57(3-4), 320-329. doi: 10.1002/ajcp.12057

Riessman, C. K. (2008). Narrative methods for the human sciences. Thousand Oaks, CA: Sage. doi: 10.1075/ni.18.1.13ada

Saldana, J. (2012). The coding manual for qualitative researchers. Thousand Oaks, CA: Sage. doi: 10.1519/JSC.0b013e3181ddfd0a

Scales, P. C., Benson, P. L., \& Mannes, M. (2006). The contribution to adolescent well-being made by nonfamily adults: An examination of developmental assets as contexts and processes. Journal of Community Psychology, 34(4), 401-413. doi: 10.1002/jcop

Snider, C. L., \& Farmer, J. R. (2016). Impacts of a Southern Indiana summer camp: Adult reflections on childhood experiences. Journal of Youth Development, 11(3), 175-187. doi: $10.5195 /$ jyd.2016.470

Strauss, A. L., \& Corbin, J. M. (1998). Basics of qualitative research: Techniques and procedures for developing grounded theory (Vol. 2). Thousand Oaks, CA: Sage.

Thurber, C. A., Scanlin, M. M., Scheuler, L., \& Henderson, K. A. (2007). Youth development outcomes of the camp experience: Evidence for multidimensional growth. Journal of Youth and Adolescence, 36(3), 241-254. doi: 10.1007/s10964-006-9142-6

Vella, S. A., Oades, L. G., \& Crowe, T. P. (2013). The relationship between coach leadership, the coachathlete relationship, team success, and the positive developmental experiences of adolescent soccer players. Physical Education \& Sport Pedagogy, 18(5), 549-561. doi: 10.1080/17408989.2012.726976

Yoon, J. S. (2002). Teacher characteristics as predictors of teacher-student relationships: Stress, negative affect, and self-efficacy. Social Behavior and Personality, 30(5), 485-493. doi: 10.2224/sbp.2002.30.5.485 